\title{
Differential Method of Thin Layer for Retaining Wall Active Earth Pressure and Its Distribution under Seismic Condition
}

\author{
Li-Min XU, Yong SUN
}

Key Laboratory of Karst Environment and Geological Hazard Prevention of Ministry of Education, Guizhou University, Guiyang 550003, China

xulimin02010@163.com, sunyong00188@163.com

Keywords: Earthquake, Retaining Wall, Active Earth Pressure, Filling by Laye.

\begin{abstract}
This paper studies the calculation of active earth pressure of retaining structure under the condition of earthquake. By comparing with the Rankine theory and Coulomb theory, we get a very good result. The analytical solutions of the previous works are for single homogeneous isotropic filling .but in this paper, the calculation formula and method are suitable for the calculating of active earth pressure of retaining wall which is for different multilayer nature filling. In this paper, the conclusion is the same with the conclusion of literature before, namely the point position of passive earth pressure is lower than the position of Rankine theory and Coulomb theory, which should also be noticed.
\end{abstract}

\section{Introduction}

Since 1920s, a lot of scholars at home and broad have carryed on a lot of research about Retaining wall active earth pressure and its distribution under seismic condition [1-5], which most about theory research and model test, put forward early and up to now still widely used is the Mononobe - Okabe theory, since then a number of seismic earth pressure calculation theory was developed based on the theory. M-O theory was based on Coulomb theory, which was see as the model of earth pressure calculation [2-9].This paper use the method of pseudo static and horizontal layer analysis method, get differential soil studies intensity distribution formula the point position of total earth pressure, rupture angle calculation formula of soil of homogeneous clayey soil retaining structure active earth pressure under seismic condition. $[2,3]$

\section{The Analysis Model}

\section{Pseudo-static Analysis}

The calculation process, the basic assumptions and horizontal and vertical earthquake force of the pseudo-static analysis are the same to [3].

\section{Analysis the Model}

As shown in figure 1, the height of retaining wall $H$,wall back Angle $\alpha$, the filling wall surface behind wall i $\beta$. Cohesive force behind wall $c_{i}$, internal friction angle ${ }^{\phi_{i}}$, cohesive force between soil wall $c_{i}^{\prime}$, external friction angle between soil wall $\phi_{i}^{\prime}$, when the rigid wall behind retaining wall move deviate from the filling behind the wall and reaches a certain level, the filling behind the wall will move along $\overline{B C}$ which course the wall heel and cause $\theta$ to the vertical direction. We assume that the sliding surface $\overline{B C}$ is a plane. Then we see the soil pressure caused by the soil wedge $\overline{A B C}$.

\section{The Theoretical Derivation}

\section{Basic Assumption}

(1)The Filling wall is layered cohesive soils. 
(2)The soil wedge Fracture surface $\overline{B C}$ is a plane.

(3)The soil wedge $A B C$ 's movement was seen as a whole rigid body.

(4)The infinitesimal body the front and bottom $\overline{e d}$ and $\overline{f g}$ respective by resultant force $q_{i}$ and $q_{i+1}$, their direction is vertical.

(5) $c_{i}$ and $c_{i}^{\prime}$ along $\overline{B C}$ and $\overline{A B}$ distributed uniformly.

\section{The Basic Formula of the Layered Soil}

The geometrical relationship and power relations of the infinitesimal body are the same to [3].

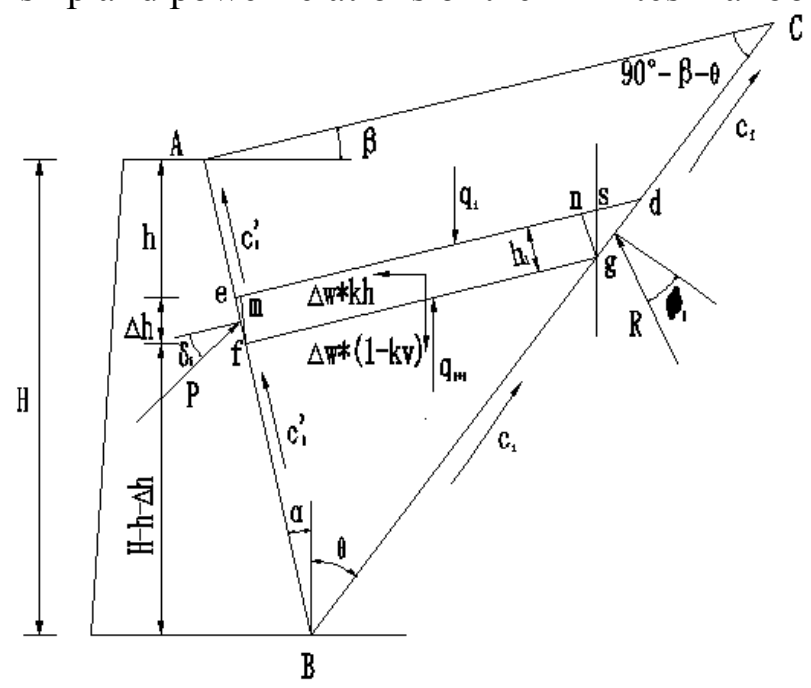

Fig. 1 A Layered Model of Active Earth Pressure

\section{Equation of Forces}

Equation of forces of horizontal direction:

$$
P_{a} \cdot \overline{e f} \cdot \cos \left(\alpha+\delta_{i}\right)-K_{h} \cdot \Delta w-c_{i}^{\prime} \cdot \overline{e f} \cdot \sin \alpha+c_{i} \cdot \overline{g d} \cdot \sin \theta-R \cdot \overline{g d} \cdot \cos \left(\theta+\phi_{i}\right)=0
$$

Equation of forces of vertical direction:

$$
P_{a} \cdot \overline{e f} \cdot \sin \left(\alpha+\delta_{i}\right)-q_{i}+q_{i+1}-\left(1-K_{v}\right) \cdot \Delta w+c_{i}^{\prime} \cdot \overline{e f} \cdot \cos \alpha+c_{i} \cdot \overline{g d} \cdot \cos \theta+R \cdot \overline{g d} \cdot \sin \left(\theta+\phi_{i}\right)=0
$$

Moment equilibrium equations:

$$
\begin{aligned}
& -P_{a} \cdot \overline{e f} \cdot \cos \left(\alpha+\delta_{i}\right) \cdot \frac{\overline{e d}+\overline{f g}}{2} \cdot \sin \beta+P_{a} \cdot \overline{e f} \cdot \sin \left(\alpha+\delta_{i}\right) \cdot \frac{\overline{e d}+\overline{f g}}{2} \cdot \cos \beta \\
& +c_{i}^{\prime} \cdot \overline{e f} \cdot \sin \alpha \cdot \frac{\overline{e d}+\overline{f g}}{2} \cdot \sin \beta+c_{i}^{\prime} \cdot \overline{e f} \cdot \cos \alpha \cdot \frac{\overline{e d}+\overline{f g}}{2} \cdot \cos \beta
\end{aligned}
$$

\section{Computation Process}

Starting from the top, $q=q_{0}=10 \mathrm{kp}_{a}, q_{0}$ is in the midpoint of $\overline{A C}, q_{i}$ and $q_{i+1}$ are in the midpoint of $\overline{e d}$ and $\overline{f g}$.

We can get:

$$
\mathrm{p}_{\mathrm{a}}=-\frac{c_{i}^{\prime} \cdot i_{1}}{i_{2}}-\frac{c_{i} \cdot i_{3}}{i_{4}}-q_{i} \cdot \frac{i_{5}}{i_{2}}-\Delta w \cdot K_{h} \frac{i_{6}}{i_{2} \cdot \Delta h}-\Delta w \cdot\left(1-K_{v}\right) \cdot \frac{i_{7}}{i_{2} \cdot \Delta h}
$$




$$
\begin{aligned}
& q_{\mathrm{i}+1}=\frac{-\mathrm{p}_{a} \cdot \Delta h \cdot \sin \left(\alpha+\delta_{i}+\theta+\varphi_{i}\right)-\mathrm{c}_{i}^{\prime} \cdot \Delta h \cdot \cos \left(\alpha+\theta+\varphi_{i}\right)}{\cos \alpha \cdot \cos \left(\theta+\varphi_{i}\right)}+q_{\mathrm{i}}+\left(1-\mathrm{K}_{\mathrm{v}}\right) \cdot \Delta \mathrm{w}_{\mathrm{i}} \\
& -\frac{\mathrm{c}_{i} \cdot \Delta h \cdot \cos (\alpha-\beta) \cdot \cos \varphi_{i}}{\cos \alpha \cdot \cos (\theta+\beta) \cdot \cos \left(\theta+\varphi_{i}\right)}+\frac{\Delta \mathrm{w} \cdot \mathrm{K}_{\mathrm{h}} \cdot \sin \left(\theta+\varphi_{i}\right)}{\cos \left(\theta+\varphi_{i}\right)} \\
& \mathrm{i}_{1}=(2 \mathrm{H}-2 \mathrm{~h}-\Delta \mathrm{h}) \cdot \sin (\theta+\alpha) \cdot \cos (\alpha-\beta) \cdot \cos \left(\theta+\varphi_{i}\right) \\
& -(\mathrm{H}-\mathrm{h}-\Delta \mathrm{h}) \cdot \sin (\theta+\alpha) \cdot \cos \beta \cdot \cos \left(\alpha+\theta+\varphi_{i}\right)-\Delta \mathrm{h} \cdot \cos (\alpha-\beta) \cdot \sin \theta \cdot \cos \left(\alpha+\theta+\varphi_{i}\right) \\
& \mathrm{i}_{2}=(2 \mathrm{H}-2 \mathrm{~h}-\Delta \mathrm{h}) \cdot \sin (\theta+\alpha) \cdot \sin \left(\alpha+\delta_{i}-\beta\right) \cdot \cos \left(\theta+\varphi_{i}\right) \\
& -(\mathrm{H}-\mathrm{h}-\Delta \mathrm{h}) \cdot \sin (\theta+\alpha) \cdot \cos \beta \cdot \sin \left(\alpha+\delta_{i}+\theta+\varphi_{i}\right)-\Delta \mathrm{h} \cdot \cos (\alpha-\beta) \cdot \sin \theta \cdot \sin \left(\alpha+\delta_{i}+\theta+\varphi_{i}\right) \\
& \mathrm{i}_{3}=\cos (\alpha-\beta) \cdot \cos \varphi_{i} \cdot[(\mathrm{H}-\mathrm{h}-\Delta \mathrm{h}) \cdot \sin (\theta+\alpha) \cdot \cos \beta+\Delta h \cdot \cos (\alpha-\beta) \cdot \sin \theta] \\
& \mathrm{i}_{4}=(2 \mathrm{H}-2 \mathrm{~h}-\Delta \mathrm{h}) \cdot \sin (\theta+\alpha) \cdot \sin \left(\alpha+\delta_{i}-\beta\right) \cdot \cos \left(\theta+\varphi_{i}\right) \cdot \cos (\theta+\beta)-(\mathrm{H}-\mathrm{h}-\Delta \mathrm{h}) \\
& \cdot \sin (\theta+\alpha) \cdot \cos \beta \cdot \sin \left(\alpha+\delta_{i}+\theta+\varphi_{i}\right) \cdot \cos (\theta+\beta)-\Delta \mathrm{h} \cdot \cos (\alpha-\beta) \cdot \sin \theta \cdot \sin \left(\alpha+\delta_{i}+\theta+\varphi_{i}\right) \cdot \cos (\theta+\beta) ; \\
& \mathrm{i}_{5}=\cos \alpha \cdot \cos \left(\theta+\varphi_{i}\right) \cdot[2 \cdot \cos (\alpha-\beta) \cdot \sin \theta-\sin (\theta+\alpha) \cdot \cos \beta] \\
& \mathrm{i}_{6}=(\mathrm{H}-\mathrm{h}-\Delta \mathrm{h}) \cdot \sin (\theta+\alpha) \cdot \cos \beta \cdot \sin \left(\theta+\varphi_{i}\right) \cdot \cos \alpha \\
& +\Delta h \cdot \cos (\alpha-\beta) \cdot \sin \theta \cdot \sin (\theta+\alpha) \cdot \cos \alpha+2 \cdot \mathrm{x} \cdot \tan \beta \cdot \cos { }^{2} \alpha \cdot \cos (\theta+\beta) \cdot \cos \left(\theta+\varphi_{i}\right) \\
& \mathrm{i}_{7}=(\mathrm{H}-\mathrm{h}-\Delta \mathrm{h}) \cdot \sin (\theta+\alpha) \cdot \cos \beta \cdot \cos \left(\theta+\varphi_{i}\right) \cdot \cos \alpha \\
& +\Delta \mathrm{h} \cdot \cos (\alpha-\beta) \cdot \sin \theta \cdot \cos \alpha \cdot \cos \left(\theta+\varphi_{i}\right)-2 \cdot \mathrm{x} \cdot \cos { }^{2} \alpha \cdot \cos (\theta+\beta) \cdot \cos \left(\theta+\varphi_{i}\right)
\end{aligned}
$$

\section{Compute Instance}

Calculation example reference Chen Xizhe tsinghua university "soil mechanics and foundation" and "retaining wall calculation handbook", and we can get that the conclusion in this passage is the same with the conclusion of literature Rankine theory and Coulomb theory.

(1) The conclusion in this passage is the same with the conclusion of Rankine theory

[1] The question is $H=6.0 \mathrm{~m}, \alpha=0^{\circ}, \beta=0^{\circ}, \delta=0^{\circ} \cdot \gamma=18 K N / \mathrm{m}^{3}, \varphi=30^{\circ}$, how to get the active earth pressure?

We let $c=0, c^{\prime}=0, k_{h}=0, k_{v}=0, q_{0}=0, \Delta h=0.25 \mathrm{~m}$, from the Rankine theory, we can get $\theta=45^{\circ}-\frac{1}{2} \varphi=30^{\circ}$, the Calculation results see the table below.

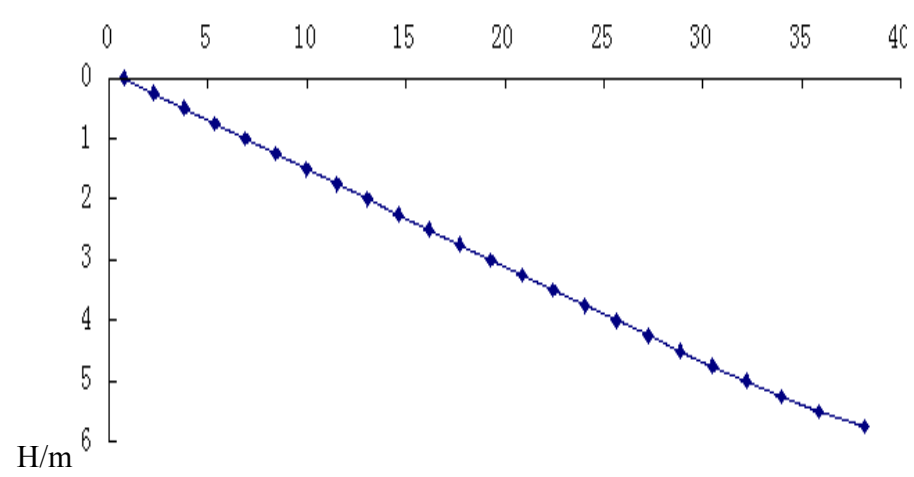

Fig.2 Active Soil Pressure Distribution of Different Clayey Soils under Seismic Condition 
Tab.1 Calculation Results by Layers of Active Soil Pressure

\begin{tabular}{cccccc}
\hline$i$ & $d w_{i}(K N)$ & $p_{a i}(K N / m) / m$ & $q_{i+1}(K N)$ & $\Delta h(m)$ & $h_{i}(m)$ \\
1 & 15.5884 & 0.7553 & 15.2613 & 0.25 & 0.00 \\
2 & 14.9389 & 2.2879 & 29.2095 & 0.25 & 0.25 \\
3 & 14.2894 & 3.8220 & 41.8439 & 0.25 & 0.50 \\
4 & 13.6399 & 5.3577 & 53.1638 & 0.25 & 0.75 \\
5 & 12.9903 & 6.8952 & 63.1684 & 0.25 & 1.00 \\
6 & 12.3408 & 8.4347 & 71.8569 & 0.25 & 1.25 \\
7 & 11.6913 & 9.9763 & 79.2283 & 0.25 & 1.50 \\
8 & 11.0418 & 11.5205 & 85.2815 & 0.25 & 1.75 \\
9 & 10.3923 & 13.0673 & 90.0154 & 0.25 & 2.00 \\
10 & 9.7427 & 14.6173 & 93.4286 & 0.25 & 2.25 \\
11 & 9.0932 & 16.1709 & 95.5196 & 0.25 & 2.50 \\
12 & 8.4437 & 17.7286 & 96.2865 & 0.25 & 2.75 \\
13 & 7.7942 & 19.2912 & 95.7273 & 0.25 & 3.00 \\
14 & 7.1447 & 20.8595 & 93.8401 & 0.25 & 3.25 \\
15 & 6.4951 & 22.4346 & 90.6208 & 0.25 & 3.50 \\
16 & 5.8456 & 24.0198 & 86.0727 & 0.25 & 3.75 \\
17 & 5.1961 & 25.6137 & 80.1777 & 0.25 & 4.00 \\
18 & 4.5466 & 27.2210 & 72.9372 & 0.25 & 4.25 \\
19 & 3.8971 & 28.8462 & 64.3435 & 0.25 & 4.50 \\
20 & 3.2475 & 30.4967 & 54.3856 & 0.25 & 4.75 \\
21 & 2.5980 & 32.1852 & 43.0470 & 0.25 & 5.00 \\
22 & 1.9485 & 33.9375 & 30.3001 & 0.25 & 5.25 \\
23 & 1.2990 & 35.8208 & 16.0802 & 0.25 & 5.50 \\
24 & 0.6495 & 38.1356 & 0.2165 & 0.25 & 5.75 \\
total & -- & 449.4955 & -- & -- & -- \\
\hline
\end{tabular}

active soil pressure distribution(MPa)

We can get the active pressure from table 1:

$\mathrm{P}_{\mathrm{a} 1}=449.4955 \times 0.25=112.3737 \mathrm{KN} / \mathrm{m}$,

We can get the active pressure from Rankine theory

$$
\begin{aligned}
& \mathrm{P}_{\mathrm{a} 2}=\frac{1}{2} \cdot \gamma \cdot H^{2} \cdot k_{a}=\frac{1}{2} \cdot \gamma \cdot H^{2} \cdot \tan ^{2}\left(45^{\circ}-\frac{1}{2} \varphi\right)=\frac{1}{2} \times 18 \times 6^{2} \times 0.34=110.16 K N / m \\
& \frac{\mathrm{P}_{\mathrm{a} 1}-\mathrm{P}_{\mathrm{a} 2}}{\mathrm{P}_{\mathrm{a} 2}}=\frac{112.3737-110.16}{110.16}=2.01 \%,
\end{aligned}
$$

The result of table 1 is agreed well with the Rankine theory.

As: $Z_{0 a}=\frac{\sum_{i=1}^{n} p_{a i} \cdot \frac{\Delta h_{i}}{\cos \alpha} \cdot\left[H-\left(\Delta h_{1}+\Delta h_{2}+\cdots+\Delta h_{i-1}+0.5 \cdot \Delta h_{i}\right)\right]}{\sum_{i=1}^{n} p_{a i}}$

Earth pressure resultant force point height $Z_{0 a} \approx 0.3528 H$,a little higher than $\frac{1}{3} H$.

(2)The conclusion in this passage is the same with the conclusion of Coulomb theory 
[2]The question is $H=6.0 \mathrm{~m}, \alpha=10^{\circ}, \beta=10^{\circ}, \delta=20^{\circ} \cdot \gamma=18 \mathrm{KN} / \mathrm{m}^{3}, \varphi=30^{\circ}$, how to get the active earth pressure?

We let $c=0, c^{\prime}=0, k_{h}=0, k_{v}=0, q_{0}=0, \Delta h=0.25 \mathrm{~m}$, we can get $\theta=45^{\circ}-\frac{\phi}{2}=45^{\circ}-\frac{14^{\circ}}{2}=38^{\circ}$, the Calculation results see the table below:

Tab. 2 Calculation Results by Layers of Active Soil Pressure

\begin{tabular}{cccccc}
\hline$i$ & $d w_{i}(K N)$ & $p_{a i}(K N / m) / m$ & $q_{i+1}(K N)$ & $\Delta h(m)$ & $h_{i}(m)$ \\
1 & 30.9188 & 0.8991 & 30.3154 & 0.25 & 0.00 \\
2 & 29.6305 & 2.7207 & 58.1201 & 0.25 & 0.25 \\
3 & 28.3422 & 4.5506 & 83.4086 & 0.25 & 0.50 \\
4 & 27.0539 & 6.3894 & 106.1748 & 0.25 & 0.75 \\
5 & 25.7657 & 8.2381 & 126.4121 & 0.25 & 1.00 \\
6 & 24.4774 & 10.0978 & 144.1131 & 0.25 & 1.25 \\
7 & 23.1891 & 11.9699 & 159.2696 & 0.25 & 1.50 \\
8 & 21.9008 & 13.8557 & 171.8722 & 0.25 & 1.75 \\
9 & 20.6125 & 15.7572 & 181.9106 & 0.25 & 2.00 \\
0 & 19.3242 & 17.6764 & 189.3728 & 0.25 & 2.25 \\
11 & 18.0359 & 19.6159 & 194.2451 & 0.25 & 2.50 \\
12 & 16.7477 & 21.5792 & 196.5117 & 0.25 & 2.75 \\
13 & 15.4594 & 23.5702 & 196.1539 & 0.25 & 3.00 \\
14 & 14.1711 & 25.5942 & 193.1495 & 0.25 & 3.25 \\
15 & 12.8828 & 27.6581 & 187.4718 & 0.25 & 3.50 \\
16 & 11.5945 & 29.7711 & 179.0879 & 0.25 & 3.75 \\
17 & 10.3062 & 31.9461 & 167.9561 & 0.25 & 4.00 \\
18 & 9.0179 & 34.2015 & 154.0225 & 0.25 & 4.25 \\
19 & 7.7297 & 36.5654 & 137.2143 & 0.25 & 4.50 \\
20 & 6.4414 & 39.0832 & 117.4282 & 0.25 & 4.75 \\
21 & 5.1531 & 41.8356 & 94.5068 & 0.25 & 5.00 \\
22 & 3.8648 & 44.9882 & 68.1815 & 0.25 & 5.25 \\
23 & 2.5765 & 48.9692 & 37.8964 & 0.25 & 5.50 \\
24 & 1.2882 & 55.6666 & 1.8286 & 0.25 & 5.75 \\
total & -- & 573.1994 & --- & -- & - \\
\hline active soil pressure distribution $(\mathrm{MPa})$ & & & &
\end{tabular}

active soil pressure distribution (MPa)

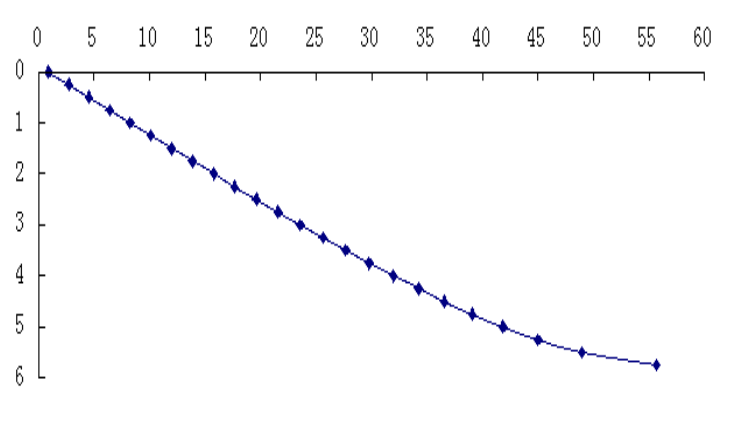

Fig.3 Active Soil Pressure Distribution of Different Clayey Soils under Seismic Condition

We can get the active pressure from table 2:

$$
P_{a 1}=573.1944 \times 0.25=143.299 \mathrm{kN} / \mathrm{m}
$$


We can get the active pressure from Coulomb theory:

$$
\begin{aligned}
& P_{\mathrm{a} 2}=\frac{1}{2} \cdot \gamma \cdot H^{2} \cdot K_{a}=\frac{1}{2} \times 18 \times 6^{2} \times 0.46=149.04 K N / m \\
& \frac{\mathrm{P}_{\mathrm{a} 2}-\mathrm{P}_{\mathrm{a} 1}}{\mathrm{P}_{\mathrm{a} 2}}=\frac{149.04-143.299}{149.04}=3.85 \%,
\end{aligned}
$$

The result of table 1 is agreed well with the Coulomb theory.

As: $\quad Z_{0 a}=\frac{\sum_{i=1}^{n} p_{a i} \cdot \frac{\Delta h_{i}}{\cos \delta}\left[H-\left(\Delta h+\cdots++h \Delta 0 . \${ }_{i} h_{1}\right.\right.}{\sum_{i=1}^{n} p_{a i}}$

Earth pressure resultant force point height $Z_{0 a} \approx 0.3475 H$, a little higher than $\frac{1}{3} H$

\section{Conclusion}

(1)This paper studies the calculation of passive earth pressure of retaining structure under the condition of earthquake. Assume that every layer $\Delta \mathrm{h}_{i}$ only transfer force downward $q_{i}$, there is no friction between the layers, that is the horizontal seismic force fully borne by retaining wall.

(2)The calculation formula and method are suitable for the calculating of active earth pressure of retaining wall which is for different multilayer nature filling .

(3)The conclusion is the same with the conclusion of literature Rankine theory and Coulomb theory.

(4)The point position of active earth pressure is higher than the position of Rankine theory and Coulomb theory, which should also be noticed,because the rigidity of the retaining wall problem is beneficial.

\section{References}

[1]Wang Weizhang.The new theory's formula and test verify to the earth pressure distribution behind retaining wall[J].South Central Traffic Technology, 1995, (2):70-73.

[2]Lin Yuliang, Yang Guolin, Zhao Lianheng. Active earth pressure of cohesive soil behind retaining walls under seismic condition [J].Rock and Soil Mechanics, vol.32 No.8, 2011.

[3]Sun Yong.Unified solution of seismin active earth pressure and its distribution on a retaining wall [J].Rock and Soil Mechanics, 2012, 33(1): 255-261.

[4]Yang Jian.Active earth pressure of inclined retaining walls under seismic condition [J].Chinese Journal of Geotechnical Engineering, 2009, 31(9):1391-1397.

[5]Wang Liqiang.Distribution of seismic soil presssure on a retaining wall[J].China Harbour Engineering, 2007,(5):1-5.

[6]Steedman R S,Zeng X,The influence of phase on the calculation of pseudo-static earth pressure on a retaining wall[J].Geotechnique ,1990,40(1):103-112.Vol.8, 311-317.

[7]Yang Jian.Passive earth pressure of inclined retaining walls under seismic condition [J].Chinese Journal of Geotechnical Engineering, 2009, 31(9):1391-1397.

[8]Lu Kunlin.Preliminary study of active earth pressure under nonlimit state[J].Rock and Soil Mechanics,2010,31(2):615-619. 